Problembereiche die Landwirtschaft, das Gesundheitswesen, Wasserressourcen und Städtebau. Im Gegensatz zur Option, die Emissionen von Treibhausgasen zu reduzieren, wurde das Thema Adaption in der gesellschaftlichen Debatte in Großbritannien bislang eher ausgeklammert, mit seiner wissenschaftlichen Bearbeitung wird gerade erst begonnen.

POST empfiehlt, zur Abschätzung der Auswirkungen von Klimaänderungen auf die genannten sensiblen Bereiche ein sog. Integrated Assessment durchzuführen, das Treibhausgas-Szenarios, Klima und Impaktmodelle miteinander koppelt und zudem die Wechselwirkungen zwischen den einzelnen Bereichen berücksichtigt und eventuelle Reaktionen auf den Klimawandel miteinbezieht. Einem "Stakeholder"-geführten Ansatz, wobei die jeweilige Problemstellung von den Entscheidungsträgern und nicht den Wissenschaftlern erfolgen soll, wird dabei der Vorzug gegeben. Diese auch für das UKCIP geplante Vorgehensweise soll die unmittelbare Berücksichtigung der Untersuchungsergebnisse im politischen Entscheidungsprozess ermöglichen.

Angesichts fehlender konkreter Adaptionsszenarien, muß sich POST auf die Ausarbeitung einer "route-map for adaptation" beschränken. Ausgehend von einer kontinuierlichen Beobachtung von Indikatoren des Klimawandels (Monitoring) und der Feststellung signifikanter Änderungen, beispielsweise durch Überschreitung vorgegebener Grenzwerte, sollte es möglich sein, "Trajektorien" des zukünftigen Verlaufs dieser Änderungen anzugeben und daraus eventuell nötige Adaptionsmaßnahmen abzuleiten. Um dieses Ideengerüst mit Leben zu erfüllen, wird allerdings nach POST noch einiges an gesellschaftlicher und insbesondere wissenschaftlicher Diskussion erforderlich sein, auch über so grundsätzliche Themen, ob der zu erwartende Klimawandel gute oder schlechte Auswirkungen haben wird und ob überhaupt derzeit eine Notwendigkeit für irgendwelche Adaptionsmaßnahmen gesehen wird.

Die Studie kann bezogen werden über: The Parliamentary Bookshop, 12 Bridge Street, London SW1A 2JX, Tel.: + 44 (0) 171-2193890, Fax: + 44 (0) 171-219-3866.

》

\section{VDI-Tagung "Wertprobleme der Technikbewertung"}

\section{Tagungsbericht von Ulrich Riehm, ITAS}

Die Vorgeschichte der VDI-Richtlinie 3780 "Technikbewertung - Begriffe und Grundlagen" reicht bis in die 70er Jahre zurück. 1989 erschien dann der sogenannte "Gründruck", der Entwurf zur Richtlinie, und 1991 wurde die Richtlinie in der endgültigen Fassung veröffentlicht. Die Statuten des VDI sehen vor, daß Richtlinien nach fünf Jahren auf ihre Gültigkeit zu überprüfen sind, gegebenenfalls überarbeitet oder zurückgezogen werden müssen. $\mathrm{Zu}$ diesem Zweck veranstaltete der VDI-Ausschuß "Grundlagen der Technikbewertung", der die Richtlinie erarbeitet hatte, eine Tagung und legte dort unter dem Titel "Aktualität der Technikbewertung. Erträge und Perspektiven der Richtlinie VDI 3780" als VDI Report 29 seine Bilanz vor. Während in dem Band, der hier nicht weiter besprochen werden soll, von den Autoren Rapp, Ropohl, Hubig und Brennecke die Erfahrungen mit der Richtlinie und die Diskussion um die Richtlinie aufgearbeitet wird eine Überarbeitung oder Neufassung schien nicht sinnvoll - konzentrierte man sich in der Tagung auf das "Wertproblem der Technikbewertung". Neun Referenten und eine Referentin gestalteten die insgesamt acht Vorträge, die sich insbesondere durch eine breite Themenvielfalt auszeichneten. Etwa 40 Teilnehmer waren nach Stuttgart ins VDI-Haus gekommen und trugen durch eine lebhafte Diskussion, für die ausreichend Zeit einkalkuliert wurde, zum Gelingen dieser Tagung bei.

Im ersten Vortrag von Klaus Peter Wagner (TU München, in Vertretung von Ralf Reichwald) ging es um "Technikbewertung am Beispiel der Telekooperation". Wagner berichtete über die Entwicklung und Erprobung eines Verfahrens zur Beurteilung einer "erweiterten Wirtschaftlichkeit". Zur Unterstützung der betrieblichen Entscheidungsprozesse werden unterschiedlich bewertete Lösungen mittels Software visualisiert.

Ebenfalls um die Rolle, die Softwaresysteme in der Generierung von Lösungen und bei der Bewertung spielen können, ging es im Vortrag von Günter Deweß (Amt für Umwelt- 
schutz, Leipzig). Der Anwendungsbereich war hier die Verkehrsplanung, konkret der geplante Südring der Autobahn um Leipzig. Neben aller Problematik der Indikatorenbildung und mathematischen Modellierung wurde auch hier deutlich, daß der Computereinsatz seine Rolle insbesondere in der Planungsdiskussion mit Bürgern und Verbänden hat, wenn alternative Vorschläge (oder alternative Bewertungen) schnell auf ihre Konsequenzen hin durchgerechnet werden können.

Wieder einen ganz anderen Technologiebereich behandelte Wolfgang Weimer-Jehlen (Akademie für Technikfolgenabschätzung, Stuttgart). Er berichtete über ein Projekt der Akademie aus den Jahren 1993-1996 zur "Klimaverträglichen Energieversorung in BadenWürttemberg". Ausgehend von dem Ziel einer Reduzierung der $\mathrm{CO}_{2}$-Emissionen um $25 \%$ wurden drei Szenarien entwickelt, die diese Vorgabe erfüllten. Die Variante "weiter machen wie bisher" würde allerdings nicht zum Erfolg führen; eine Entscheidung zwischen den drei Szenarien müsse also gefällt werden. Die Szenarien dienten auch dazu, die auftretenden Wertkonflikte bearbeitbar zu machen.

Jörg Schlaich und Anette Bögle (Universität Stuttgart) stellten sich dem Problem, wie Ingenieurbauten - konkret Brücken - einer ganzheitlicheren Betrachtungsweise unterzogen werden können. In einer eindrucksvollen Diaserie wurde - gemäß dem Diktat eines zu eng verstandenen Ökonomiedenkens - der Verfall der Kultur des Brückenbaus dokumentiert. Um dem entgegenzuwirken, wurde ein umfassendes Bewertungsschema, mit den fünf Hauptkriterien Tragwerk und Gestalt, Funktionalität, Wohlbefinden, Umweltverträglichkeit, Wirtschaftlichkeit, entwickelt. Damit will man den Baubehörden Gründe an die Hand geben, auch vermeintlich teurere Lösungen auswählen zu können.

Über Bewertungsfragen im Projekt "Technikfolgen Chlorchemie" berichtete Heimfrid Wolff (Prognos, Basel). In diesem schon Anfang der 90er Jahre durchgeführten Projekt scheiterte man damit, die VDI-Richtlinie direkt umzusetzen, gewann dann aber aus dem Wertekatalog der Richtlinie doch Anregungen für die Generierung alternativer Lösungen.

Dem aktuellen Thema "Nachhaltigkeit" widmete sich Hans Diefenbacher (Forschungsstätte der Evangelischen Studiengemeinschaft,
Heidelberg). Er verglich die Indikatoren (Ziele), die in Nachhaltigkeitsprojekten in Städten und Gemeinden (u.a. Heidelberg, Kassel, Luxemburg) entwickelt wurden, mit denjenigen der VDI-Richtlinie und stellte dabei einen überraschenden Grad an Übereinstimmung her, aber auch einige grundlegende Unterschiede (z.B. beim Ziel "internationale Konkurrenzfähigkeit" der VDI-Richtlinie).

Über eine ungewöhnliche Kooperation berichteten Carsten Henschel (Hoechst, Frankfurt) und Christian Hochfeld (Öko-Institut, Darmstadt). Gemeinsam arbeitet man an einem Verfahren zur Bewertung von neuen Produkten unter Gesichtspunkten einer nachhaltigen Entwicklung.

Den Schlußvortrag hielt Hariolf Grupp (Fraunhofer Institut für Systemtechnik und Innovationsforschung, Karlsruhe) zum Thema "interkulturelle Unterschiede der Technikbewertung in Europa und Japan". Eine seiner Thesen war, daß man in Japan von einem formulierten Bedarf ausgehe und dafür dann nach technologischen Lösungen suche, während man in Europa eher Technologien entwickele, deren Anwendungspektrum erst noch erschlossen werden müsse.

Die Vorträge, die hier nur kurz angesprochen werden konnten, sollen in einer Publikation des VDI veröffentlicht werden. Die angestrebte Diskussion um die Erfahrungen mit der Anwendung der Richtlinie spiegelte sich nur in ganz wenigen Vorträgen (am ehesten bei Wolff und Dieffenbacher). Die Wert- oder Bewertungsproblematik bildete den roten Faden durch die Vielfalt der in den Vorträgen angesprochenen Technologiebereiche. Dabei wurde deutlich, daß der Einsatz von Bewertungsverfahren weniger dazu dient, "optimale" Lösungen zu finden. Mindestens genauso wichtig ist die diskursive Funktion in (partizipativen) Bewertungsverfahren. Dabei scheint es durchaus nicht ungewöhnlich, daß in einem iterativen Prozeß die zunächst formulierten Werte nach einer ersten Lösung revidiert werden, gefundene Lösung und Ausgangswerte also in einem wechselseitigen Verhältnis stehen. Eine weitere Funktion scheint darin zu bestehen, mit Hilfe von (neuen) Bewertungsverfahren die in bestimmten Bereichen vorherrschenden Werte verändern zu wollen. Damit wird die Bewertungsfrage aus dem Kontext einer engen Ent- 
scheidungslogik zwischen alternativen Lösungen herausgelöst.

Der VDI-Report 29 kann zu einem Preis von DM 18,-- bezogen werden vom Verein Deutscher Ingenieure, VDI-Hauptgruppe, GrafRecke-Straße 84, D-40239 Düsseldorf, Fax: 0211/6214-148, e-mail: brennecke@vdi.de. Der Tagungsband wird im Verlag edition sigma erscheinen.

》

\section{Bericht über den 5. Weltkon- greß "Intelligent Transport Systems" in Seoul, Korea}

\section{Konferenzbericht von Günter Halbritter und Torsten Fleischer, ITAS}

Die international wohl bedeutendste Jahrestagung zum Einsatz von Informations- und Kommunikationstechniken (IuK-Techniken) im Verkehr, der 5. Weltkongreß "Intelligent Transport Systems" vom 12.-16. Oktober in Seoul/Korea, war durch eine Vielzahl interessanter Einzelergebnisse in diesem aus seiner ersten stürmischen Entwicklungsphase herausgetretenen Technikbereich geprägt, wenngleich herausragende Ereignisse fehlten. Insgesamt stellte auch diese ITS-Konferenz, wie auch bereits ihre Vorgänger, mit drei Plenary Sessions, 12 Executive Sessions mit 72 Beiträgen, 28 Special Sessions mit 140 Beiträgen und 104 Technical Sessions mit 520 Beiträgen eine Mammutveranstaltung dar. Mehr als die Hälfte der technischen Konferenzbeiträge stammte aus Korea und dem übrigen asiatisch-pazifischen Raum (hier v.a. aus Japan). Zeitgleich mit der Konferenz fand eine Ausstellung zum praktischen Einsatz von IuK-Techniken im Verkehrsbereich statt. Diese wurde hauptsächlich von asiatischen Ausstellern bestritten. Im Unterschied zur Vorgängerveranstaltung in Berlin, bei der alle Regionen in großem Umfang vertreten waren, war die Präsenz amerikanischer und europäischer Hersteller auf dieser Ausstellung eher gering. Die deutsche Industrie wurde lediglich durch die Unternehmen Bosch-Signalbau Huber und Dr. Seufert GmbH Karlsruhe repräsentiert.

In diesem Bericht sollen einige Einschätzungen zu dieser technisch-wissenschaftlichen
Großveranstaltung gegeben werden. Einschränkend muß bemerkt werden, daß eine solch umfangreiche Veranstaltung mit bis zu zwölf parallel laufenden Sitzungen auch von zwei Konferenzteilnehmern, die zudem noch eigenen Vortragsverpflichtungen nachkommen mußten, nicht zu leisten war. Neben einer allgemeinen, notwendigerweise subjektiven Einschätzung zu Ablauf und inhaltlicher Schwerpunktsetzung des Gesamtkongresses, beschränkt sich die Darstellung auf einige ausgewählte Sitzungen, wobei als Auswahlkriterium der Einsatz von IuK-Techniken in Ballungsräumen diente. Vorliegender Bericht entspricht somit nicht dem Anspruch auf eine umfassende und objektive Berichterstattung zum Kongreß.

Die Tagung stand insgesamt ganz im Zeichen des Straßenverkehrs, öffentliche Verkehrsträger spielten nur eine untergeordnete Rolle. Weiterhin war die Tagung sehr von technischen Beiträgen geprägt, die zum großen Teil aus dem asiatisch-pazifischen Raum stammten. Beiträge, die sich mit der Umsetzung dieser Techniken beschäftigten, waren deutlich in der Minderheit, entsprechend auch Wirkungs- und Folgenanalysen dieser Techniken. Diesbezügliche Ansätze waren zwar in manchen Beiträgen - vor allem von europäischen und australischen(!), in geringerem Umfang auch von nordamerikanischen Referenten - zu finden, zeigten aber auch hier häufig die hinlänglich bekannten Defizite bezüglich empirischer Fundierung und Übertragbarkeit. Festzustellen ist, daß Problembewußtsein in bezug auf Forschungsbedarf $\mathrm{zu}$ Wirkungen und Folgen von IuK-Techniken im Verkehr hauptsächlich von Vertretern aus Ländern mit vergleichsweise geringer (oder nur punktueller) industrieller Aktivität in der Verkehrstelematik signalisiert wurde (etwa die skandinavischen Länder und die Niederlande, aber auch Australien und Neuseeland), während seitens der Vertreter der großen "Forschungsnationen" eher das Herausstellen von Erwartungen und Hoffnungen dominierte.

Inhaltlich prägend für die Konferenz waren unserer Einschätzung nach letztlich die Beiträge aus dem anglo-amerikanischen Raum, obwohl der Anteil technischer Beiträge aus dem Bereich der europäischen Union fast doppelt so hoch war wie der aus den USA (140 zu 79). Ein Grund für diese Einschätzung ist sicherlich die konzeptionelle Klarheit, die insbesondere die 